\title{
Análisis de la Frecuencia Cardiaca relacionada con las variables de altura y frecuencia en el Levantamiento de cargas
}

\author{
Analysis of Heart Rate related to height and frequency \\ of manual lifting
}

\author{
Luis A. Saavedra-Robinson ${ }^{1}$ \\ Yenny A. Paredes-Astudillo² \\ Leonardo Quintana ${ }^{1}$ \\ ${ }^{1}$ Pontificia Universidad Javeriana, Facultad de ingeniería, Departamento de ingeniería industrial \\ Bogotá, Colombia. \\ 2Institución Universitaria Politécnico Grancolombiano, Departmento de ingeniería industrial. Bogotá, \\ Colombia.
}

Fechas · Dates

Recibido: 2020.08 .05

Aceptado: 2020.11.13

Publicado: 2021.01.21
Correspondencia · Corresponding Author

Luis A. Saavedra-Robinson.

E-mail: I.saavedra@javeriana.edu.co. 


\section{Resumen}

Objetivo: Analizar los efectos de las variables altura vertical y la frecuencia de levantamiento sobre la frecuencia cardiaca en trabajadores en la tarea de levantamiento de cargas.

Método: Diseño experimental factorial con bloques, considerando como variables independientes la altura vertical y la frecuencia de levantamiento y la dependiente la frecuencia cardiaca. El experimento se desarrolló con 20 trabajadores entre 18 y 40 años con al menos seis meses de experiencia en tareas de almacén y empaque en una empresa de productos lácteos. Se calculó el coeficiente FRIMAT para determinar la carga fisiológica de la tarea.

Resultados: Existe un efecto significativo sobre la respuesta de la frecuencia cardiaca debido a la interacción de la frecuencia y la altura vertical del levantamiento. Además, basados en el coeficiente FRIMAT, se obtuvo una calificación de "carga de trabajo mínima" para el $85 \%$ de los tratamientos, y en un tratamiento, se presentó una calificación de "algo difícil".

Conclusiones: La altura y frecuencia de levantamiento no actúan de manera independiente y tienen un efecto sobre la carga fisiológica del trabajador en el levantamiento manual de cargas manifestada en la frecuencia cardiaca.

Palabras clave: Ergonomía; Frecuencia Cardíaca; Carga de Trabajo; Dolor Musculoesquelético.

\section{Abstract}

Objective: To analyze the effects of vertical height and lifting frequency on the physiological response in workers during lifting of loads.

Methods: Factorial experimental design with blocks, considering vertical height and lifting frequency as independent variables; the dependent variable was defined as the heart rate. The experiment was conducted in 20 workers between the ages of 18 and 40 years with at least six months of experience in warehouse and packaging tasks at a dairy products company. We calculated the FRIMAT coefficient to determine the physiological load of the task.

Results: We found a significant effect on the heart rate response due to the interaction of the rate and the vertical height of the lift. Based on the FRIMAT coefficient, we obtained a rating of "minimal workload" for $85 \%$ of the treatments; for one treatment there was a rating of "somewhat difficult."

Conclusions: Height and frequency of lift do not act separately and have an effect on the physiological load of the worker during manual lifting, reflected by changes in the heart rate.

Keywords: ergonomics; heart rate; workload; musculoskeletal pain. 


\section{Introducción}

Desde que las primeras industrias de manufactura iniciaron sus operaciones, el manejo manual de materiales (MMM) siempre ha sido una tarea que ha involucrado el uso intensivo de mano de obra debido al dinamismo de la tarea. Esta actividad ha causado muchas lesiones y una carga económica de más de 100 mil millones de dólares americanos por año en el caso de los Estados Unidos ${ }^{(1)}$. Esta carga económica incluye el costo médico y no médico, así como los costos directos y los costos intangibles ${ }^{(2)}$.

A pesar del incremento en el uso de la automatización para realizar estas actividades, en algunos sistemas actuales de producción, persisten acciones manuales en la recogida, el trasporte y el levantamiento de cargas. Estas acciones implican esfuerzos biomecánicos que comúnmente conllevan en la aparición de trastornos musculoesqueléticos, pero también, una gran demanda física por tratarse de un trabajo extremadamente dinámico. De hecho, estudios anteriores han examinado los beneficios de las nuevas tecnologías y concluyeron que el uso de equipos como los exoesqueletos durante una tarea de elevación, podría aumentar la frecuencia cardíaca media del individuo(3).

Diferentes estudios han demostrado que el consumo de oxígeno, la frecuencia cardíaca y el gasto de energía son apropiados para evaluar las demandas metabólicas generales del trabajo donde el levantamiento de carga implica caminar, cargar y otras tareas de manipulación manual(4,5,6). Por ejemplo, estudios recientes utilizaron la Reserva de la Frecuencia Cardíaca (\% HRR) como parámetro y han demostrado que, si bien la actividad física en tiempo libre de trabajo promueve la salud, la actividad física laboral puede tener consecuencias negativas para la salud ${ }^{(7)}$. Los trabajadores que realizan altos niveles de actividad física en el trabajo muestran un mayor riesgo de ausencia por enfermedad a largo plazo(8). e inclusive trastornos cardiovasculares ${ }^{(9)}$.

Cuando se menciona el levantamiento directo en el $\mathrm{MMM}^{(10,11)}$, la ecuación revisada del National Institute for Occupational Safety and Health (NIOSH) (Revised NIOSH Lifting Equation siglas en inglés RNLE) es una de las herramientas más utilizadas para evaluar el riesgo ergonómico de la tarea de elevación. Este método incluye algunos parámetros como frecuencia, asimetría, desplazamiento de la carga, pero no implica una demanda metabólica en el límite de peso recomendado (Recommended Weight Limit siglas en inglés RWL). Nuestro grupo inició algunos estudios para analizar diferentes parámetros sobre el levantamiento manual. Un primer artículo fue publicado en $2012^{(12)}$ sobre la determinación del límite de peso máximo aceptable (Maximum acceptable weight of lift siglas en inglés MAWL) por parte de los trabajadores varones adultos, sin embargo, no fue incluido el consumo metabólico para dicho análisis. El objetivo de este segundo estudio es analizar la frecuencia cardiaca (FC) durante las tareas de elevación de cargas en determinadas condiciones de altura y frecuencia considerando, además, la incomodidad de las partes del cuerpo y determinar bajo el coeficiente FRIMAT si existe un nivel de esfuerzo fisiológico considerable en esta actividad. 


\section{Métodos}

Se realizó este estudio con diseño experimental. Un experimento permite ver más de cerca la causa y el efecto al revisar qué resultados que ocurren cuando se manipulan factores particulares en el proceso de experimentación.

\section{Sujetos}

La población objeto de estudio corresponde a trabajadores activos de una empresa colombiana productora y distribuidora de productos lácteos, quienes desempeñaban sus labores en el centro de distribución. Los parámetros de inclusión en el estudio son: trabajadores de sexo masculino, entre 18 y 40 años, con experiencia mínima en el cargo de seis meses. Los convocados aceptaron voluntariamente participar en el estudio firmando un consentimiento de aprobación.

Para el cálculo del tamaño de la muestra se usó la ecuación mostrada a continuación, con un nivel de confianza del 95\% y tomando como base la variabilidad del estudio publicado por Saavedra-Robinson y colaboradores en el primer estudio en el 2012 ${ }^{(12)}$, dónde $\sigma=1.96$.

$$
n=\frac{\left(Z_{\frac{\alpha}{2}}\right)^{2} * \sigma^{2} * N}{\left(e^{2} *(N-1)+\left(Z_{\frac{\alpha}{2}}\right)^{2} * \sigma^{2}\right)}
$$

Se determinó un tamaño de muestra mínima de 14.45 15, sin embargo, se logró contar con 20 participantes interesados en el estudio, por lo cual se trabajó con este tamaño de muestra.

\section{Procedimiento e instrumentos}

El estudio se desarrolló en un espacio de 12×4.5 metros de área dentro de la compañía, adaptado especialmente para el desarrollo de la prueba en condiciones simuladas. En este espacio se dispusieron de 70 canastas plásticas de 30×40×25 $\mathrm{cm}$ y 1100 bolsas de leche de $900 \mathrm{~g}$ con las cuales se llenaron las canastas para el desarrollo del experimento, esto con el fin de mantener lo más cercano a la realidad las condiciones de experimentación y una báscula digital para controlar el peso de levantamiento.

Previamente a la puesta en marcha de la etapa de recolección de datos se realizaron 2 pruebas piloto que permitieron corregir la disposición de los elementos en la prueba, y definir previamente el plan para garantizar la aleatoriedad en la toma de datos; posteriormente después de tomar las medidas correctivas y ya seleccionados los participantes, se realizó en la primera fase en encuentro informativo, en el cual se le explicaron a los participantes el objeto del estudio, las instrucciones para la realización de las pruebas y el cronograma de cada uno de los participantes ajustado según sus turnos laborales. De igual manera el área de salud ocupacional 
de la compañía realizó una valoración médica previamente para garantizar que la participación en el experimento no tendría afectaciones sobre el estado de salud de las personas.

Cada participante asistió al sitio de experimentación en 11 días, contando un día para cada uno de los 9 tratamientos, el espacio de información y valoración. Ya estando el participante en el sitio de prueba, se le otorgó un tiempo de 5 minutos para realizar ejercicios de estiramiento y posteriormente se colocó sobre el pecho del sensor de frecuencia cardiaca y el reloj polar en la muñeca para dar inicio con la aplicación del tratamiento correspondiente.

El observador mientras tanto se encargaba de identificar el tratamiento y disponer las condiciones para cada prueba ubicando en posición al participante tomando fotografías y verificando así las condiciones de levantamiento establecidas previamente en el entrenamiento.

\section{Diseño del experimento}

El estudio incluyó el muestreo y análisis de la FC, que estaba sujeta a un diseño de experimento basado en dos factores: la altura y la frecuencia de levantamiento; tres niveles por factor. Teniendo en cuenta que este estudio fue aplicado a varios trabajadores, todos los tratamientos se aplicaron a cada participante, proponiendo a los trabajadores como una variable de bloque en el experimento. Los trabajadores levantaron una canasta de $30 \times 40 \times 25 \mathrm{~cm}$ cuando el peso de la carga se mantuvo constante durante el estudio.

Factor Frecuencia: el número de veces que un individuo realiza una tarea durante un intervalo (en este caso durante un minuto); en fracciones de tiempo iguales. Los tres niveles de frecuencia fueron dos veces, cuatro veces y seis veces por minuto. Factor de altura: se refiere a las diferentes alturas a las que los colaboradores tuvieron que llevar a cabo la carga. Los tres niveles de altura fueron desde el piso hasta un poco más debajo de los nudillos, en este estudio, esta altura corresponde a las siguientes distancias ( 0 a $52.5 \mathrm{~cm}$ ); desde los nudillos hasta un poco más abajo el hombro $(52.5$ a $125 \mathrm{~cm}$ ) y desde los hombros hasta el alcance máximo (125 a $175 \mathrm{~cm})$.

La Figura 1, presenta las combinaciones de niveles que dan lugar a cada uno de los tratamientos del experimento. 


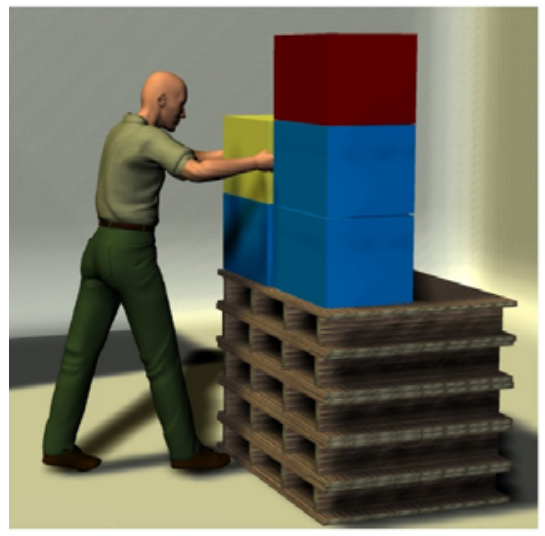

\begin{tabular}{|c|c|c|c|}
\hline Factor & Nivel & \multicolumn{2}{|c|}{ Descripción } \\
\hline \multirow{3}{*}{$\begin{array}{c}\text { A } \\
\text { (Frecuencia) }\end{array}$} & -1 & \multicolumn{2}{|c|}{2 veces por minuto, es decir cada 30 segundo } \\
\hline & 0 & \multicolumn{2}{|c|}{4 veces por minuto, es decir cada 15 segundos } \\
\hline & 1 & \multicolumn{2}{|c|}{6 veces por minuto, es decir cada 10 segundos } \\
\hline \multirow{3}{*}{$\begin{array}{c}\text { B } \\
\text { (Altura) }\end{array}$} & -1 & \multicolumn{2}{|c|}{ Del suelo a los nudillos (0 a $52.5 \mathrm{~cm}$ ). } \\
\hline & 0 & \multicolumn{2}{|c|}{ De nudillos a hombros (52.5 a $125 \mathrm{~cm}$ ) } \\
\hline & 1 & \multicolumn{2}{|c|}{ De hombros a altura máxima (125 to $175 \mathrm{~cm}$ ) } \\
\hline Bloque & Nivel & \multicolumn{2}{|c|}{ Descripción } \\
\hline Participante & $1-20$ & \multicolumn{2}{|c|}{ Trabajadores } \\
\hline Tratamiento & & Altura & Frecuencia \\
\hline $\mathbf{I}$ & \multicolumn{2}{|c|}{\begin{tabular}{|l|l|} 
Suelo - Nudillo \\
\end{tabular}} & \multirow{3}{*}{2 veces por minuto } \\
\hline II & \multicolumn{2}{|c|}{ Nudillo - Hombro } & \\
\hline III & \multicolumn{2}{|c|}{ Hombro - Altura máxima } & \\
\hline IV & \multicolumn{2}{|c|}{ Suelo - Nudillo } & \multirow{3}{*}{4 veces por minuto } \\
\hline V & \multicolumn{2}{|c|}{ Nudillo - Hombro } & \\
\hline VI & \multicolumn{2}{|c|}{ Hombro - Altura máxima } & \\
\hline VII & \multicolumn{2}{|c|}{ Suelo - Nudillo } & \multirow{3}{*}{6 veces por minuto } \\
\hline VIII & \multicolumn{2}{|c|}{ Nudillo - Hombro } & \\
\hline IX & \multicolumn{2}{|c|}{ Hombro - Altura máxima } & \\
\hline
\end{tabular}

Figura 1: Resumen del diseño experimental utilizado en la investigación.

La Figura 1 ilustra también las condiciones de levantamiento de la prueba y se muestra un ejemplo de la descripción de los tratamientos aplicados, en la izquierda la caja amarilla representa el nivel 0 del factor B (Altura desde los nudillos hasta los hombros), la caja roja el nivel 1 del factor B (Altura desde los hombros hasta alcance máximo). Se definió un experimento bloqueado con dos factores cada uno con tres niveles. Los nueve (9) tratamientos se replicaron tres veces y se aplicaron de manera aleatoria en cada bloque (trabajador), la cantidad de registros obtenidos fue $\mathrm{N}=540$, donde $\mathrm{N}=9$ tratamientos $\times 20$ trabajadores $\times 3$ repeticiones. El modelo lineal de los efectos se presenta a continuación.

$$
Y_{i j l k}=\mu+\alpha_{i}+\beta_{j}+\tau_{l}+\varepsilon_{i j l k}+\alpha \beta_{i j}
$$

$Y_{i j \mid k}$ : es el valor observado cuando el factor A está en el nivel i, el factor B está en el nivel j.

el bloque en el nivel I y corresponde a la réplica k.

$\mu$ : es la media global.

$a_{i}$ : es el efecto del factor A en el nivel i.

$\beta_{i}$ : es el efecto del factor $B$ en el nivel $\mathrm{j}$.

$\tau_{i}$ es el efecto del bloque en el nivel I.

$\varepsilon_{i j k}$ : es el componente el error aleatorio.

$a \beta_{i j}$ : es el efecto de la interacción del factor $A$ en el nivel i y el factor $B$ en el nivel j.

\section{Valoración de la carga física mediante el monitoreo de la frecuen- cia cardiaca}

La utilidad de la frecuencia cardiaca como método de evaluación ha sido demostrada por múltiples estudios y ha estado descrita desde los aspectos cualitativos 
y cuantitativos. En el caso cuantitativo, existen propuestas como las de Chamoux y FRIMAT ${ }^{(13)}$ que permiten medir el nivel de esfuerzo basado en el monitoreo de la FC con equipos de lectura directa (monitor de frecuencia cardiaca). Para evaluar el esfuerzo de la tarea en este estudio, se realizó el criterio FRIMAT, este criterio consiste en asignar coeficientes de coacción (de 1 a 6) a los diferentes criterios cardíacos. La suma de todos los coeficientes se utiliza para calificar la tarea, clasificándola de acuerdo con su nivel de penosidad y costo cardíaco.

Para la Relación entre la incomodidad de las partes del cuerpo y los tratamientos del estudio se utilizó una modificación del cuestionario nórdico estandarizado para recopilar la incomodidad del cuerpo en cada tratamiento(14). Este cuestionario ya está reconocido y validado y se usa generalmente para detectar síntomas o molestias en la sección del cuerpo, como los hombros, el cuello, la espalda y otras extremidades ${ }^{(15)}$.

\section{Análisis de datos}

Los resultados de frecuencia cardiaca experimentales fueron analizados a través de un Análisis de Varianza - ANOVA, implementado en SPSS ${ }^{\text {. El }}$ El objetivo es identificar los efectos significativos de los factores evaluados sobre la variable de respuesta. Se verificaron los supuestos de normalidad por medio de la prueba de Kolmogórov-Smirnov con un nivel de significancia del $5 \%$ junto con un gráfico PPPlot. Con respecto a la homogeneidad aplicó un gráfico de predichos vs residuos. Finalmente, el supuesto de independencia se aseguró con la aplicación de los tratamientos a cada una de las personas (bloques) de manera aleatoria.

\section{Resultados}

La tabla 1 muestra el resultado obtenido por el Análisis de Varianza - ANOVA. El $p$-valor $<0.05$, permite rechazar la hipótesis nula (la cual indica que no existe efecto), según los resultados existe efecto significativo sobre la respuesta de la FC dado por la frecuencia de levantamientos, la altura del levantamiento, y la interacción de los dos factores. Adicionalmente tal como se presumía el efecto del sujeto que realiza la prueba (bloque) también resulto ser significativo.

Tabla 1: Respuesta obtenida de la Frecuencia Cardiaca.

\begin{tabular}{lrrrrr}
\hline Fuente & Suma de cuadrado & gl & Cuadrado medio & F & P-valor \\
\hline Bloque (Trabajador) & 93824.361 & 19 & 4938.124 & 54.056 & .000 \\
A (Frecuencia) & 1464.115 & 2 & 732.057 & 8.014 & .000 \\
B (Altura) & 741.070 & 2 & 370.535 & 4.056 & .018 \\
AxB & 1363.874 & 4 & 340.969 & 3.732 & .005 \\
Error & 46772.422 & 512 & 91.352 & & \\
Total & 144165.843 & 539 & & &
\end{tabular}


El gráfico PP-Plot (Figura 2a) y la prueba de Kolmogórov-Smirnov fue aplicado para verificar si, los residuos tipificados siguen una distribución normal, esta prueba permitió aceptar la hipótesis de normalidad con valor de $p=0.2$. El gráfico de predichos versus residuos fue usado para probar la homogeneidad de las varianzas (Figura 2b).

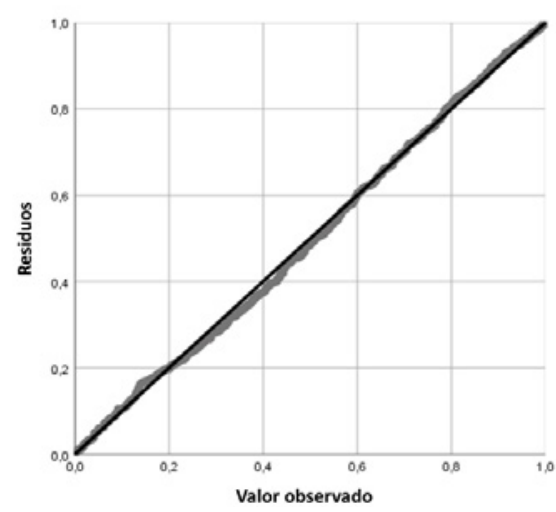

Figura 2a: PP-Plot.

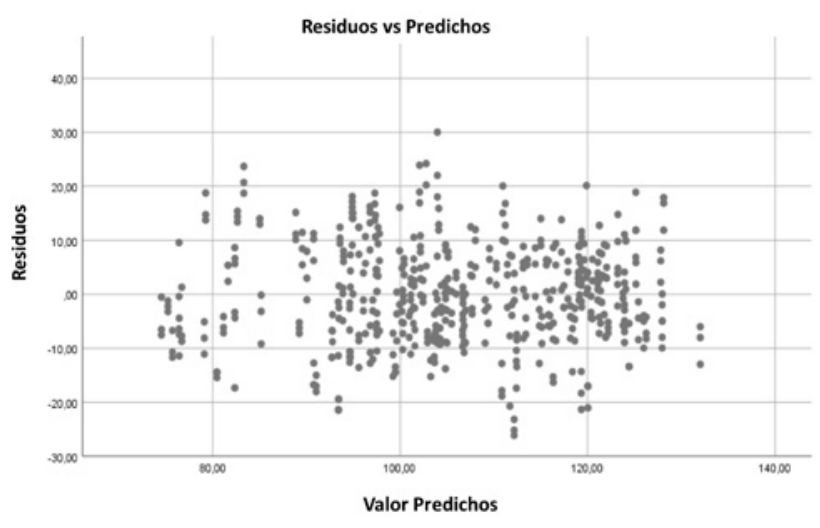

Figura 2b: Predichos vs Residuos.

Figura 2: Verificación de normalidad estadística en la respuesta de la Frecuencia Cardiaca.

El análisis muestra que existe evidencia estadística la cual indica que el factor A, B, y su interacción tiene un efecto sobre la variable de respuesta. El valor más alto de la media del ritmo cardíaco (112 bpm) fue obtenido como resultado de la interacción $A B$, cuando el factor $A$ (frecuencia) se encuentran en el nivel uno (1), 6 veces por minuto y el factor $B$ (altura) se encuentra en el nivel uno (1), desde los hombros hasta el alcance máximo (Figura 3).

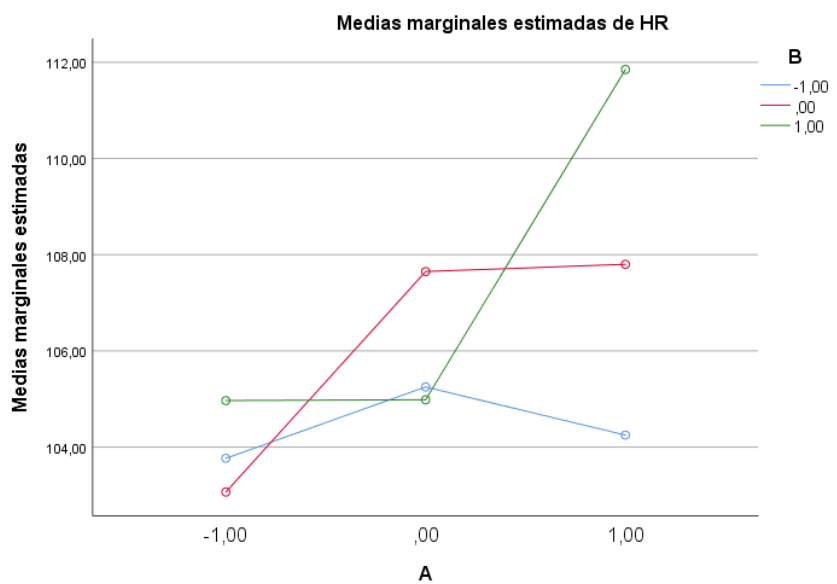

Figura 3: Efecto de la interacción del Factor A (frecuencia) y B (altura) sobre el ritmo cardíaco. 
Igualmente, para el factor B (Altura) en los niveles (-1) y (0) muestra un decrecimiento en la media del ritmo cardiaco, lo cual corrobora los supuestos planteados por $\mathrm{NIOSH}$, el cual indica que la altura deseable para el levantamiento debe estar entre las caderas y los hombros. Por otro lado, altura superior a los hombros genera un incremento en la media del ritmo de cardiaco.

\section{Determinación del nivel de esfuerzo para la respuesta de la FC basado en el coeficiente FRIMAT}

Como se observa en la Figura 4, los valores obtenidos bajo el coeficiente FRIMAT establece que el trabajo fue categorizado en un 70\% como de "carga física mínima", pero es importante afirmar que el 30\% restante estaba entre las categorías "Suave" y "Muy Suave".

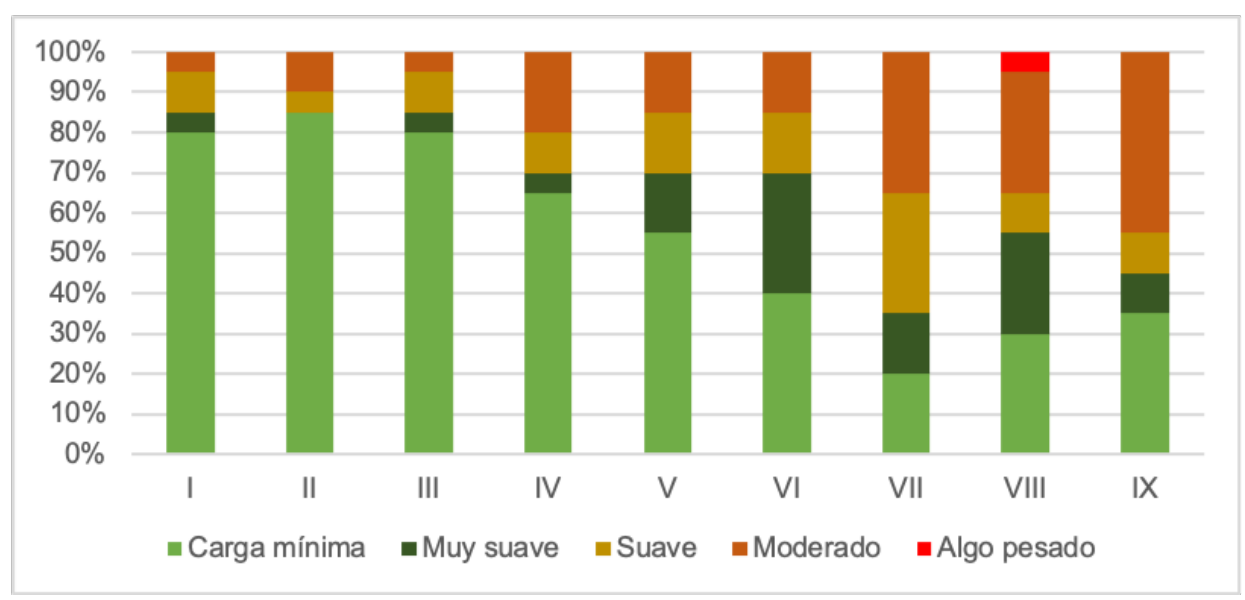

Figura 4: Resultados de la evaluación de la carga física bajo el coeficiente FRIMAT.

Dentro de estos valores, la variable del costo cardíaco relativo (CCR\%) fue el factor de influencia para los participantes que no obtuvieron un valor más bajo y, por lo tanto, todos encontraron un nivel de carga física mínima. Para los primeros tres tratamientos, se obtuvo una calificación de "carga mínima" de trabajo para una proporción que varía entre el $80 \%$ al $85 \%$. En los siguientes tratamientos, la proporción de participantes que calificaron la tarea como carga mínima disminuye y aumenta la calificación "moderada". En el tratamiento VIII, se presentó una calificación de "algo pesado". 


\section{Relación entre la incomodidad de las partes del cuerpo y los trata- mientos del estudio}
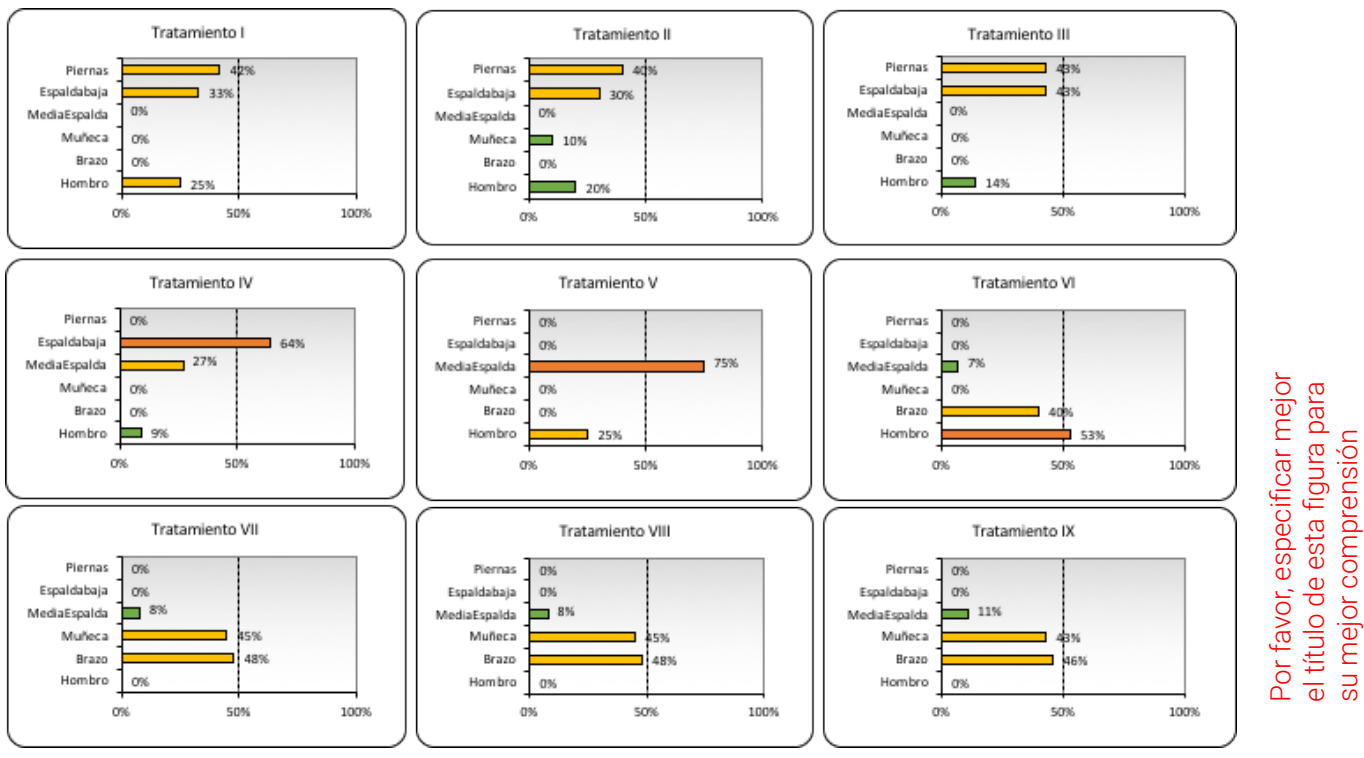

Figura 5: Porcentaje de Incomodidad en las partes del cuerpo de acuerdo a cada tratamiento del experimento.

Como se puede observar en la figura 5, en los tratamientos I, II y III, la espalda baja y las piernas son las partes del cuerpo más afectadas al levantar una carga desde el piso hasta los nudillos. En el caso de los tratamientos VII, VIII y IX, las áreas más afectadas del cuerpo fueron los brazos y las muñecas para levantar una carga desde los hombros al máximo, independientemente de la frecuencia de levantamiento de estos tres últimos tratamientos.

\section{Discusión}

El control de la frecuencia de levantamiento de manera independiente mostró que esta variable influencia el nivel del ritmo cardiaco de manera directa, por tal razón es posible concluir que el incremento en el número de repeticiones por periodo de tiempo en una actividad de levantamiento conduce a un incremento en la demanda fisiológica. En particular, el estudio evidencia que el tratamiento que considera la interacción entre frecuencia de 6 levantamientos por minuto y alturas superiores a los hombros obtuvo la media superior del ritmo cardiaco 112bpm, siendo consistente con el análisis de varianza que rectifica el efecto de la interacción. Considerando que las características individuales de los participantes, su acondicionamiento físico y entrenamiento podría ser una fuente importante de variabilidad de los datos, se encontró pertinente el uso de un diseño con bloques, resultando el efecto de los bloques significativo sobre la variable de respuesta. 
Al igual que Elfeituri y Taboun(16) quienes muestran que la frecuencia de levantamiento es uno de los parámetros determinantes en el límite de peso (RWL) recomendado por NIOSH y donde según sus resultados, un cambio en la frecuencia al pasar de 3 a 6 levantamientos por minuto genera una reducción del 51\% del límite de peso recomendado. Por otro lado, los diseños factoriales se utilizan ampliamente en experimentos que involucran varios factores para identificar los efectos de interacción de diferentes factores en el patrón de respuesta. En un estudio desarrollado por Maiti y Bagchi(17), se compararon los efectos de tres factores de levantamientos (Frecuencia, altura vertical y peso levantado) sobre la frecuencia cardiaca, a diferencia del presente estudio, se generaron varios tratamientos haciendo la combinación de estos tres factores, sin embargo, en el tratamiento que se compara las variables altura y frecuencia, la frecuencia como variable de respuesta osciló entre $100 \mathrm{bpm}$ y $110 \mathrm{bpm}$ para un peso levantado de $15 \mathrm{Kg}$, siendo muy similar a los $112 \mathrm{bpm}$ para $18 \mathrm{Kg}$ que se presentaron en el presente estudio.

Adicionalmente, a diferencia de los estudios similares presentados, este estudio proporciona una evaluación de molestias sentidas, donde se observaron que los participantes durante el desarrollo de los tratamientos IV, $\vee$ y VI manifestaron molestias que afectaron más del $50 \%$ de la población estudio. Es importante destacar que la molestia y su ubicación sobre el cuerpo fue modificándose en cuanto el tratamiento fue aumentando la altura vertical y la frecuencia del levantamiento, donde en los primero tres tratamientos (2 veces por minuto) el dolor fue en piernas y espalda baja, en los subsiguientes tratamientos fue espalda media y espalda baja y en tres últimos tratamientos fue a la altura de brazos y muñecas que es cuando más la carga está alejada del cuerpo y son las extremidades superiores quienes intervienen directamente con la acción del torque para mantener el sistema en reposo y no dejar caer el producto que se está levantando, aspecto que ha sido ampliamente estudiado por el multiplicador horizontal de la ecuación de NIOSH.

Dentro de las limitaciones del presente estudio, se encuentra la técnica de lectura directa utilizada para la recolección de información, la cual, a pesar de presentar un nivel de precisión considerable, impide obtener un tamaño de muestra mayor a comparación de metodologías como la observación o autorreportes. En cuanto al equipo investigador, una limitante del estudio fue el acceso a las personas participantes, pues al tratarse de un estudio de caso, el acceso a las instalaciones de la empresa fue muy restringida debido a las condiciones de bioseguridad derivado de la pandemia COVID-19, esto conllevó a ajustar el estudio a los protocolos de bioseguridad emitidos por la empresa participante que derivaron en demoras para la obtención de la información.

Los resultados de este estudio muestran que los factores que influyen en el levantamiento no actúan de manera aislada y que sus efectos no influyen de manera directa en la carga fisiológica de un trabajador, sin embargo, la interacción de estos factores si pueden tener consecuencias fisiológicas. Ante ello, se recomienda para trabajos futuros incluir el factor peso levantado del objeto como una variable a considerar, pues estudios anteriores ${ }^{(12,17)}$. han considerado este parámetro, algunos relacionado con la respuesta de la frecuencia cardiaca y otros no, pero que, 
en definitiva, puede potencialmente tener efectos sobre la respuesta fisiológica de un trabajador. Estos estudios permiten conocer de manera asertiva los factores que influyen en el diseño del puesto de trabajo y así procurar estaciones de trabajo acordes a las respuestas fisiológicas del trabajador, que, por el dinamismo de la tarea, pueda estar colocando en riesgo su salud. Finalmente, dentro de los estudios futuros, se recomienda involucrar no solo los levantamientos de cargas sino también el transporte de las cargas, esto debido a que, por las condiciones del estudio, este factor de riesgo no fue evaluado, pero sería de interés involucrar la carga acumulada, pues debido a su dinamismo, puede tener un efecto en el rendimiento fisiológico del participante.

\section{Bibliografía}

1. Yin P, Yang L, Wang C, Qu S. Effects of wearable power assist device on low back fatigue during repetitive lifting tasks. Clin Biomech. 2019;70:59-65.

2. Dagenais S, Caro J, Haldeman S. A systematic review of low back pain cost of illness studies in the United States and internationally. Spine J. 2008; 8(1):8-20.

3. Desbrosses K, Roux T,Savescu, A. Physiological consequences of using an upper limb exoskeleton during manual handling tasks. Appl Ergon. 2018;67:211217.

4. Rohmert W, Laurig W, Philipp U, Luczak $\mathrm{H}$. Heart rate variability and work-load measurement. Ergonomics. 1973;16(1):33-44.

5. Jørgensen K. Permissible loads based on energy expenditure measurements. Ergonomics. 1985;28(1):365-369.

6. Fox RR, Lu ML, Occhipinti E, Jaeger M. Understanding outcome metrics of the revised NIOSH lifting equation. Appl Ergon. 2019;81:102897.

7. Coenen P, Korshøj M, Hallman DM, Huysmans MA, van der Beek AJ, Straker LM, et al. Differences in heart rate reserve of similar physical activities during work and in leisure time-a study among Danish blue-collar workers. Physiol Behav. 2018;186:45-51.

8. Holtermann A, Hansen JV, Burr H, Søgaard K, Sjøgaard G. The health paradox of occupational and leisure-time physical activity. Br J Sports Med. 2012;46(4):291295.

9. Li J, Loerbroks A, Angerer P. Physical activity and risk of cardiovascular disease: what does the new epidemiological evidence show?. Curr Opin Cardiol. 2013;28(5):575-583.

10. Hattori Y, Ono Y, Shimaoka M, Hiruta S, Shibata E, Ando S, et al. Effects of box weight, vertical location and symmetry on lifting capacities and ratings on category scale in Japanese female workers. Ergonomics. 2000;43(12):2031-2042.

11. Yeung SS, Genaidy AM, Deddens J, Leung PC. What is a demanding lifting job for manual handling workers in Hong Kong?. Ergonomics. 2003;46(6):574-597. 
12. Saavedra-Robinson $L A$, Quintana $L A$, Fortunato LD, Niño M. Analysis of the lifted weight including height and frequency factors for workers in Colombia. Work. 2012; 41 Suppl 1:1639-1646.

13. Frimat $P, A m p h o u x ~ M, C h a m o u x A$. Interprétation et mesure de la fréquence cardiaque. Revue de Medicine du Travail. 1988;15(4):147-165.

14. Kuorinka I, Jonsson B, Kilbom A, Vinterberg H, Biering-Sørensen F, Andersson $\mathrm{G}$, et al. Standardised Nordic questionnaires for the analysis of musculoskeletal symptoms. Appl Ergon. 1987:18(3): 233-237.

15. Palmer K, Smith G, Kellingray S, Cooper C. Repeatability and validity of an upper limb and neck discomfort questionnaire: the utility of the standardized Nordic questionnaire. Occup Med. 1999;49(3):171-175.

16. Elfeituri FE, Taboun SM. An evaluation of the NIOSH lifting equation: a psychophysical and biomechanical investigation. Int J Occup Saf Ergo. 2002;8(2):243258.

17. Maiti R, Bagchi TP. Effect of different multipliers and their interactions during manual lifting operations. International Journal of Industrial Ergonomics. 2006;36(11):991-1004. 